\title{
Cortical bone loss in patients with chronic kidney disease
}

Patients with chronic kidney disease (CKD) have an increased risk of fracture but the biochemical and microarchitectural basis of this increased risk has not been fully defined. Now, Thomas Nickolas and colleagues report that patients with CKD show rapid loss of cortical bone, which is associated with hyperparathyroidism and increased levels of bone remodelling markers.

"The advent of high resolution imaging methods that can separately quantify abnormalities in cortical and trabecular bone motivated us to study the impact of CKD on the skeleton," explains Nickolas. "We utilized traditional and novel boneimaging methods to determine the impact of the disease on the evolution of bone structure, and used a panel of biochemical markers of bone metabolism to determine the biochemical mechanisms that drive this evolution."

In their longitudinal study of 53 patients with CKD stage $2-5 \mathrm{D}$, the researchers assessed bone structure and levels of bone remodelling markers at baseline and at a

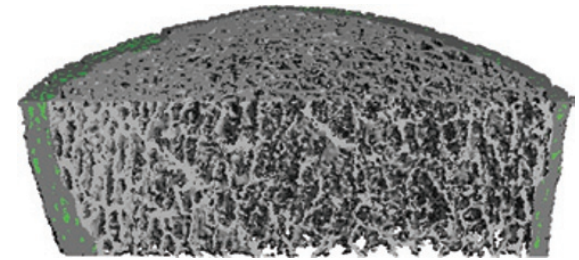

High resolution peripheral quantitative CT image showing cortical pores in the tibia of a male patient aged 66 years on dialysis. Image courtesy of T. L. Nickolas, Division of Nephrology, Columbia University Medical Center, NY, USA.

median follow-up of 1.5 years. They found that areal bone mineral density (assessed using dual energy X-ray absorptiometry) decreased by $1.3 \%$ per year at the hip and by $2.4 \%$ per year at the ultradistal radius. High resolution peripheral quantitative CT assessment showed that cortical area, density and thickness decreased and cortical porosity increased from baseline at the distal radius, and cortical density decreased and cortical porosity increased from baseline at the tibia. No significant changes in trabecular density or in the number or heterogeneity of the trabecular network at the distal radius or tibia were detected. Higher time-averaged levels of parathyroid hormone and bone remodelling markers were associated with significant decreases in cortical area and cortical thickness at the distal radius, and increased levels of bone remodelling markers were also associated with decreases in cortical density and increases in cortical porosity at the tibia.

The researchers conclude that CKD is associated with cortical bone loss, possibly as a result of hyperparathyroidism and increased bone turnover. They plan to investigate if loss of cortical bone leads to decreased bone strength in patients with $\mathrm{CKD}$, and hope to determine whether agents that suppress production of parathyroid hormone or reduce rates of bone remodelling could decrease cortical bone loss in these patients.

Ellen F. Carney

Original article Nickolas, T. L. et al. Rapid cortical bone loss in patients with chronic kidney disease. J. Bone Miner. Res. doi:10.1002/jbmr.1916 